\title{
Probability distributions in statistical ensembles with conserved charges
}

\author{
J. Cleymans* \\ UCT-CERN Research Centre and Department of Physics, University of Cape Town, Rondebosch 7701, South Africa \\ K. Redlich ${ }^{\dagger}$ and L. Turko $\ddagger$ \\ Institute of Theoretical Physics, University of Wrocław, Pl. Maksa Borna 9, 50-204 Wrocław, Poland
}

(Received 21 December 2004; published 29 April 2005)

\begin{abstract}
The probability distributions for charged particle numbers and their densities are derived in statistical ensembles with conservation laws. It is shown that if this limit is properly taken, then the canonical and grand canonical ensembles are equivalent. This equivalence is proven on the most general probability distribution level.
\end{abstract}

DOI: 10.1103/PhysRevC.71.047902

PACS number(s): 25.75.-q, 24.10.Pa, 24.60.Ky, 05.20.Gg

Macroscopic data of an equilibrium state are described by means of statistical distributions of microscopic variables specific for a given ensemble. In the application to the description of particle production in high energy particle collisions, we are generally dealing with the grand canonical ensemble with respect to the particle number [1-3]. In the ultrarelativistic situation, energy conservation and particle number are usually controlled by the temperature of the system [4].

Applications of statistical physics concepts to multiparticle production processes require, however, the implementation of internal symmetries $[5,6]$ that result in associated conservation of quantum numbers. In the grand canonical formulation (GC), conservation of quantum numbers is applied to the average and is determined by the corresponding chemical potential. On the other hand, in the canonical ensemble $(\mathrm{C})$ quantum number conservation is specifically treated.

In general, the thermodynamic quantities calculated in the GC and $\mathrm{C}$ ensembles differ. This is particularly true when dealing with small systems $[1-4,7,8]$. Since relativistic heavy ion collisions correspond to a finite volume and to a given charge value, the canonical ensemble should be used whenever the GC and C formalisms give different answers [2,8-11]. It is, however, to be expected that in the thermodynamic limit, the $\mathrm{C}$ and GC descriptions will provide the same answer for physical observables $[2,9,10]$. The thermodynamic limit is reached in large volume for a fixed density in $\mathrm{C}$, and for a fixed average density in the GC ensemble. Only in this limit can one indiscriminately use the GC or $\mathrm{C}$ descriptions. The results presented here do not rely on the system under consideration being of a relativistic nature and apply equally well to relativistic and nonrelativistic systems.

There is an essential difference in the volume dependence of observables in the GC and $\mathrm{C}$ formulations [1-3,7,9]. Consequently, in the limit when $V \rightarrow \infty$, some ratios of extensive quantities could in general converge to different

*Email address: cleymans@qgp.phy.uct.ac.za

${ }^{\dagger}$ Email address: redlich@ift.uni.wroc.pl

†Email address: turko@ift.uni.wroc.pl values in the $\mathrm{GC}$ and $\mathrm{C}$ ensembles [12]. It is thus clear that the equivalence of the two descriptions in the thermodynamic limit can be strictly established only for intensive observables [2].

In applications of statistical models to particle production in high energy collisions of elementary particles [13] and in heavy ion collisions $[1,14]$, we are always dealing with small systems. Thus, the model description of particle yields is in principle canonical with respect to the conservation laws. This is particularly evident in elementary particle and peripheral heavy ion collisions where, e.g., strangeness production is strongly suppressed due to canonical effects [11]. However, a detailed analysis of different particle yields [1,9-11,14] has shown that in central heavy ion collisions the relative error between the $\mathrm{C}$ and GC descriptions is so small that the GC approximation can be used with confidence.

Recently, an interesting observation has been made [12] that in finite systems even very small relative errors between $\mathrm{C}$ and GC results seen on the level of first particle moments, i.e., thermal particle multiplicities, do not necessarily guarantee that this is also the case for higher moments or particle fluctuations. On the contrary, for a set of thermal parameters relevant in high energy heavy ion collisions, the second moment differs substantially in the GC and $\mathrm{C}$ ensembles at finite $V$ even though the first moments agree to high accuracy. The above observation has been generalized [12] to the large volume limit with the conclusion that $\mathrm{GC}$ and $\mathrm{C}$ ensemble equivalence is violated in the thermodynamic limit on the level of particle number fluctuations.

However, a direct comparison of GC and $\mathrm{C}$ results for the average charged particle number or higher moments is only adequate for a finite system. In the thermodynamic limit, such a comparison can be only done at the level of densities. This is simply because in the large volume limit, only particle densities are finite in GC and C ensembles, whereas corresponding multiplicities are infinite in both cases. In the thermodynamic limit, the equivalence problem of $\mathrm{GC}$ and $\mathrm{C}$ formulation of the conservation laws requires intensive observables.

We show that different statistical ensembles with respect to conservation laws are exactly equivalent in the thermodynamic limit. Consequently, there is equivalence of all possible moments, relative fluctuations, scaled variance, etc. The exact 
calculations are performed in the $\mathrm{GC}$ and $\mathrm{C}$ ensembles with arbitrary values of the conserved charge.

The equivalence of canonical and grand canonical ensembles of statistical systems with conservation laws is discussed for a noninteracting relativistic gas of charged particles and antiparticles in a volume $V$ at temperature $T$. Particles and antiparticles have charges \pm 1 , respectively.

Let us consider a system with total overall charge $Q$, with $N=N_{-}$antiparticles and $N_{+}=N+Q$ particles. The requirement of the exact charge conservation in the canonical ensemble leads to the partition function [1-3]

$\mathcal{Z}_{Q}^{C}(V, T)=\operatorname{Tr}_{Q} \mathrm{e}^{-\beta \hat{H}}=\sum_{N=\max (-Q, 0)}^{\infty} \frac{z^{2 N+Q}}{N !(N+Q) !}=I_{Q}(2 z)$,

where $z$ is the one-particle partition function

$$
\begin{aligned}
z(T) & =\frac{V}{(2 \pi)^{3}} \int d^{3} p \mathrm{e}^{-\beta \sqrt{p^{2}+m^{2}}} \\
& =\frac{V}{2 \pi^{2}} T m^{2} K_{2}\left(\frac{m}{T}\right) \equiv V z_{0}(T) .
\end{aligned}
$$

$I_{Q}$ and $K_{2}$ are the modified Bessel functions [15].

To obtain the average particle multiplicity moments, one introduces, in, e.g., (1), fugacity parameters $\lambda_{+}$and $\lambda_{-}$, which are set to one in the final formulas

$$
\begin{aligned}
\mathcal{Z}_{Q}^{C}\left(V, T, \lambda_{-}, \lambda_{+}\right) & =\sum_{N=\max (-Q, 0)}^{\infty} \frac{z^{2 N+Q}}{N !(N+Q) !} \lambda_{+}^{N+Q} \lambda_{-}^{N} \\
& =\left(\frac{\lambda_{+}}{\lambda_{-}}\right)^{Q / 2} I_{Q}\left(2 z \sqrt{\lambda_{+} \lambda_{-}}\right)
\end{aligned}
$$

such that

$$
\left\langle\left. N_{ \pm}^{k}\right|_{Q} ^{C}=\left.\frac{1}{\mathcal{Z}_{Q}^{C}}\left(\lambda_{ \pm} \frac{\partial}{\partial \lambda_{ \pm}}\right)^{k} \mathcal{Z}_{Q}^{C}\left(V, T, \lambda_{-}, \lambda_{+}\right)\right|_{\lambda_{ \pm=1}} .\right.
$$

From Eqs. (3-4), it is clear that

$$
\mathcal{P}_{Q}^{C}(N, N+Q, V)=\frac{z^{2 N+Q}}{N !(N+Q) !} \frac{1}{I_{Q}(2 z)}
$$

is the probability distribution to find $N$ negatively and $N+$ $Q$ positively charged particles in a system of volume $V$, temperature $T$, and total charge $Q[16,17]$.

In the grand canonical ensemble, the charge is conserved on the average; thus the partition function

$$
\mathcal{Z}^{G C}(V, T)=\operatorname{Tr} \mathrm{e}^{-\beta \hat{H}+\gamma \hat{Q}},
$$

where $\gamma$ is chosen so as to reproduce the average charge $\langle Q\rangle$ in the system. For a noninteracting gas, the trace in the above equation can be calculated explicitly, yielding

$$
\begin{aligned}
\mathcal{Z}^{G C}\left(V, T, \gamma, \lambda_{-}, \lambda_{+}\right) & =\sum_{N_{+}=0}^{\infty} \sum_{N_{-}=0}^{\infty} \frac{\mathrm{e}^{\gamma\left(N_{+}-N_{-}\right)} \lambda_{+}^{N_{+}} \lambda_{-}^{N_{-}}}{N_{+} ! N_{-} !} z^{N_{-}+N_{+}} \\
& =\exp \left[\left(\lambda_{+} \mathrm{e}^{\gamma}+\lambda_{-} \mathrm{e}^{-\gamma}\right) z\right],
\end{aligned}
$$

where, as in Eq. (3), we have introduced auxiliary fugacities $\lambda_{ \pm}$ for particles and antiparticles. Following Eq. (4), the average number of particles and the average charge in the GC ensemble are obtained as

$$
\left\langle N_{ \pm}\right\rangle=z \exp ( \pm \gamma), \quad\langle Q\rangle=\left\langle N_{+}\right\rangle-\left\langle N_{-}\right\rangle=2 z \cosh \gamma .
$$

In terms of the total charge $Q=N_{+}-N_{-}$, the GC partition function (7) can be written as

$$
\mathcal{Z}^{G C}\left(V, T, \gamma, \lambda_{-}, \lambda_{+}\right)=\sum_{N=0}^{\infty} \sum_{Q=-N}^{\infty} \mathrm{e}^{\gamma Q} \frac{\lambda_{+}^{Q+N} \lambda_{-}^{N}}{N !(Q+N) !} z^{Q+2 N},
$$

with $N=N_{-}$being the number of negatively charged particles. Thus, the function

$$
\mathcal{P}_{\langle Q\rangle}^{G C}(N, N+Q, V)=\frac{1}{\mathcal{Z}^{G C}} \frac{z^{Q+2 N}}{N !(Q+N) !} \mathrm{e}^{\gamma Q}
$$

defines the probability distribution in a $\mathrm{GC}$ ensemble, with average charge $\langle Q\rangle$, to find a charge $Q$ with $N$ negatively charged particles. Expressing the chemical potential appearing in (10) through the corresponding average charge from (8), one finds

$$
\begin{aligned}
\mathcal{P}_{\langle Q\rangle}^{G C}(N, N+Q, V)= & \frac{z^{2 N+Q}}{N !(N+Q) !}\left[\frac{\langle Q\rangle+\sqrt{\langle Q\rangle^{2}+4 z^{2}}}{2 z}\right]^{Q} \\
& \times \mathrm{e}^{-\sqrt{\langle Q\rangle^{2}+4 z^{2}}} .
\end{aligned}
$$

All other less restricted probabilities can now be obtained directly from Eq. (11). The probability distributions $\mathcal{P}_{\langle Q\rangle}^{G C}(N, V)$ to find $N$ particles, and $\mathcal{P}_{\langle Q\rangle}^{G C}(Q, V)$ to find charge $Q$, in the volume $V$ at a given average charge $\langle Q\rangle$ are obtained from

$$
\begin{aligned}
& \mathcal{P}_{\langle Q\rangle}^{G C}(N, V)=\sum_{Q=-N}^{\infty} \mathcal{P}_{\langle Q\rangle}^{G C}(N, N+Q, V), \\
& \mathcal{P}_{\langle Q\rangle}^{G C}(Q, V)=\sum_{N=\max (-Q, 0)}^{\infty} \mathcal{P}_{\langle Q\rangle}^{G C}(N, N+Q, V) .
\end{aligned}
$$

The summations in Eqs. (12a) and (12b) can be done explicitly, yielding

$$
\begin{aligned}
\mathcal{P}_{\langle Q\rangle}^{G C}\left(N_{ \pm}, V\right)= & \frac{1}{N_{ \pm} !}\left[\frac{\sqrt{\langle Q\rangle^{2}+4 z^{2}} \pm\langle Q\rangle}{2}\right]^{N_{ \pm}} \\
& \times \exp \left[-\frac{\sqrt{\langle Q\rangle^{2}+4 z^{2}} \pm\langle Q\rangle}{2}\right] .
\end{aligned}
$$

For the charge distribution, one finds

$\mathcal{P}_{\langle Q\rangle}^{G C}(Q, V)=I_{Q}(2 z)\left[\frac{\langle Q\rangle+\sqrt{\langle Q\rangle^{2}+4 z^{2}}}{2 z}\right]^{Q} \mathrm{e}^{-\sqrt{\langle Q\rangle^{2}+4 z^{2}}}$.

The particle number probability distribution (13a) is, as expected [16,17], a Poisson distribution. The charge distribution, on the other hand, is not Poissonian due to the constraints imposed by the requirement of the exact charge conservation 
in a given sector of the GC ensemble with a fixed average charge $\langle Q\rangle$.

Earlier, we introduced a set of probability distributions in the $\mathrm{GC}$ and $\mathrm{C}$ ensembles related to the charged particle number. All these distributions are also valid for any value of the conserved charge as well as the volume of the system. Thus, they can be used to analyze the thermodynamic limit.

From (5) and (11), one finds

$$
\mathcal{P}_{\langle Q\rangle}^{G C}(N, N+Q, V)=\mathcal{P}_{Q}^{C}(N, N+Q, V) \mathcal{P}_{\langle Q\rangle}^{G C}(Q, V) .
$$

It is straightforward that in the sector of fixed charge $Q$, the corresponding particle number is distributed as in the canonical ensemble. That is why in Eq. (14) the GC probability function $\mathcal{P}_{\langle Q\rangle}^{G C}$ is just the product of the canonical particle number and the grand canonical charge distribution.

To take the thermodynamic limit in (14), one first expresses the variables $(N, Q,\langle Q\rangle)$ by means of the corresponding densities $(n, q,\langle q\rangle)$, and then one takes the limit $V \rightarrow \infty$ for fixed densities. This also requires the replacement of a discrete $\operatorname{sum}(1 / V) \sum_{N} \rightarrow \int d n$.

The essential difference between GC and $\mathrm{C}$ distributions in Eq. (14) appears through the probability function $\mathcal{P}_{\langle Q\rangle}^{G C}(Q, V)$. Thus, to study the equivalence of $\mathrm{GC}$ and $\mathrm{C}$ ensembles on the probability level in the thermodynamic limit, it is sufficient to find $\mathcal{P}_{\langle q\rangle}^{G C^{\infty}}(q)$ from

$$
\mathcal{P}_{\langle q\rangle}^{G C^{\infty}}(q)=\lim _{V \rightarrow \infty} V \mathcal{P}_{\langle q\rangle}^{G C}(q, V),
$$

where $\mathcal{P}_{\langle q\rangle}^{G C}$ is obtained from Eq. (13b) by replacing $\langle Q\rangle$ and $Q$ by $V\langle q\rangle$ and $V q$, respectively. The large volume limit in (15) is taken at fixed densities. An extra volume factor in Eq. (15) appears from the replacement of discrete by continuum variables.

The limit in Eq. (15) is obtained from the $\alpha \rightarrow \infty$ behavior of the Bessel function [15]

$$
I_{\alpha}(\alpha x) \simeq \frac{\mathrm{e}^{\alpha \sqrt{1+x^{2}}}}{\sqrt{2 \pi \alpha}\left(1+x^{2}\right)^{1 / 4}}\left[\frac{x}{1+\sqrt{1+x^{2}}}\right]^{\alpha} .
$$

Consequently, the charge density probability distribution

$$
\begin{aligned}
\mathcal{P}_{\langle q\rangle}^{G C^{\infty}}(q)=\lim _{V \rightarrow \infty} & \left\{V^{1 / 2} \frac{\mathrm{e}^{-V\left(\sqrt{\langle q\rangle^{2}+4 z_{0}^{2}}-\sqrt{q^{2}+4 z_{0}^{2}}\right)}}{\sqrt{2 \pi}\left(q^{2}+4 z_{0}^{2}\right)^{1 / 4}}\right. \\
& \left.\times\left[\frac{\langle q\rangle+\sqrt{\langle q\rangle^{2}+4 z_{0}^{2}}}{q+\sqrt{q^{2}+4 z_{0}^{2}}}\right]^{V q}\right\} .
\end{aligned}
$$

It is rather straightforward to see from Eq. (17) that the limit $V \rightarrow \infty$ does not exist as a regular function. This limit is zero for any $q \neq\langle q\rangle$ and infinity for $q=\langle q\rangle$. Let us consider, however, the thermodynamic limit in Eqs. (15) and (17) as a generalized function

$$
\mathcal{F}=\lim _{V \rightarrow \infty} V \int d q P_{\langle q\rangle}^{G C}(q, V) f(q) .
$$

The density integration in (18) is obtained through the saddlepoint method

$$
\mathcal{F}=\lim _{V \rightarrow \infty} V^{1 / 2} \int d q \mathrm{e}^{V S(q)} f(q),
$$

where the function

$$
\begin{aligned}
S(q)= & \sqrt{q^{2}+4 z_{0}^{2}}-\sqrt{\langle q\rangle^{2}+4 z_{0}^{2}}+q \log \\
& \times\left(\langle q\rangle+\sqrt{\langle q\rangle^{2}+4 z_{0}^{2}}\right)-q \log \left(q+\sqrt{q^{2}+4 z_{0}^{2}}\right) .
\end{aligned}
$$

In the large volume limit, the dominant contribution to the integral (19) is obtained as

$$
\mathcal{F}=\lim _{V \rightarrow \infty} V^{1 / 2}\left\{\sqrt{-\frac{2 \pi}{V S^{\prime \prime}\left(q_{0}\right)}} g\left(q_{0}\right) \mathrm{e}^{V S\left(q_{0}\right)}+\mathcal{O}\left(V^{-3 / 2}\right)\right\},
$$

where $q_{0}=\langle q\rangle$ is just a saddle-point such that $S^{\prime}\left(q_{0}\right)=0$. From Eq. (21), one finds

$$
\mathcal{F}=f(\langle q\rangle),
$$

which means that the charge density probability distribution (15) converges to a delta function

$$
\mathcal{P}_{\langle q\rangle}^{G C^{\infty}}(q)=\delta(q-\langle q\rangle) .
$$

The above result, together with Eq. (14), taken in the thermodynamic limit completes the proof of equivalence of the $\mathrm{GC}$ and $\mathrm{C}$ ensembles on the probability level. The probability of finding a given density of particles and antiparticles in the GC ensemble with a fixed average charge density $\langle q\rangle$ is exactly equal to the corresponding probability in the $\mathrm{C}$ ensemble if one identifies charge density $q$ in the $\mathrm{C}$ ensemble with $\langle q\rangle$. The same replacement $q \rightarrow\langle q\rangle$ is required for all intensive thermodynamic observables.

The equivalence of the grand canonical and canonical descriptions of the conservation laws has been considered in the thermodynamic limit. The problem has been studied in a relativistic gas composed only of one type of noninteracting particles and antiparticles with the constraints imposed by charge conservation. Detailed studies of the equivalence problem of canonical and grand canonical ensembles were presented on the level of probability distributions. In the thermodynamic limit, the probability distributions for particle and antiparticle densities coincide in canonical and grand canonical ensembles. Consequently, for charged particle densities, there is full equivalence of all possible scaled moments, relative fluctuations, and scaled variance in the thermodynamic limit.

We acknowledge the stimulating discussions with A. Keränen. K. R. also acknowledges fruitful discussions with R. Gavai and H. Satz. This work is partially supported by the Polish Committee for Scientific Research under Contract KBN 2 P03B 069 25, and the Polish-South African Science and Technology Cooperation Project. 
[1] P. Braun-Munzinger, K. Redlich, and J. Stachel, in Quark Gluon Plasma 3, edited by R. C. Hwa and X. N. Wang (World scientific, Singapore), nucl-th/0304013.

[2] R. Hagedorn and K. Redlich, Z. Phys. C 27, 541 (1985).

[3] J. Rafelski and M. Danos, Phys. Lett. B97, 279 (1980); B. Muller and J. Rafelski, ibid. B116, 274 (1982).

[4] F. M. Liu, K. Werner, and J. Aichelin, Phys. Rev. C 68, 024905 (2003); F. Becattini and L. Ferroni, Eur. Phys. J. C 38, 225 (2004); F. M. Liu, K. Werner, J. Aichelin, M. Bleicher, and H. Stöcker, J. Phys. G 30, S589 (2004).

[5] K. Redlich and L. Turko, Z. Phys. C 5, 201 (1980); L. Turko, Phys. Lett. B104, 153 (1981).

[6] L. Turko and J. Rafelski, Eur. Phys. J. C 18, 587 (2001).

[7] M. I. Gorenstein, S. I. Lipskikh, V. K. Petrov, and G. M. Zinovev, Phys. Lett. B123, 437 (1983); H. T. Elze, W. Greiner, and J. Rafelski, ibid. B124, 515 (1983); J. Cleymans, A. Keränen, M. Marais, and E. Suhonen, Phys. Rev. C 56, 2747 (1997); M. I. Gorenstein, M. Gazdzicki, and W. Greiner, Phys. Lett. B483, 60 (2000).

[8] J. Cleymans, K. Redlich, and E. Suhonen, Z. Phys. C 51, 137 (1991).

[9] J. Cleymans, H. Oeschler, and K. Redlich, Phys. Rev. C 59, 1663 (1999).

[10] A. Keränen and F. Becattini, Phys. Rev. C 65, 044901 (2002).

[11] J. S. Hamieh, K. Redlich, and A. Tounsi, Phys. Lett. B486, 61
(2000); J. Phys. G 27, 413 (2001); K. Redlich and A. Tounsi, Eur. Phys. J. C 24, 589 (2002).

[12] V. V. Begun, M. Gazdzicki, M. I. Gorenstein, and O. S. Zozulya, Phys. Rev. C 70, 034901 (2004); V. V. Begun, M. I. Gorenstein, A. P. Kostyuk, and O. S. Zozulya, nucl-th/0410044; V. V. Begun, M. I. Gorenstein, and O. S. Zozulya, nucl-th/0411003; A. Keränen, F. Becattini, V. V. Begun, M. I. Gorenstein, O. S. Zozulya, nucl-th/0411116.

[13] F. Becattini and U. Heinz, Z. Phys. C 76, 269 (1997).

[14] P. Braun-Munzinger, D. Magestro, K. Redlich, and J. Stachel, Phys. Lett. B518, 41 (2001); P. Braun-Munzinger, J. Stachel, J. P. Wessels, and N. Xu, ibid. B344, 43 (1995); P. Braun-Munzinger, I. Heppe, and J. Stachel, ibid. B465, 15 (1999); F. Becattini, J. Cleymans, A. Keränen, E. Suhonen, and K. Redlich, Phys. Rev. C 64, 024901 (2001).

[15] M. Abramowitz and I. A. Stegun, Handbook of Mathematical Functions (National Bureau of Standards, Washington, D.C., 1964).

[16] J. Cleymans and P. Koch, Z. Phys. C 52, 137 (1991).

[17] C. M. Ko, V. Koch, Z. W. Lin, K. Redlich, M. Stephanov, and X. N. Wang, Phys. Rev. Lett. 86, 5438 (2001); Z. W. Lin and C. M. Ko, Phys. Rev. C 64, 041901(R) (2001); S. Jeon, V. Koch, K. Redlich, and X. N. Wang, Nucl. Phys. A697, 546 (2002); K. Redlich, V. Koch, and A. Tounsi, ibid. A702, 326 (2002); K. Redlich, J. Cleymans, H. Oeschler, and A. Tounsi, Acta Phys. Pol. B 33, 1609 (2002). 